\title{
FIGO Stage IIA
}

National Cancer Institute

\section{Source}

National Cancer Institute. FIGO Stage IIA. NCI Thesaurus. Code C96253.

A FIGO stage term that applies to gynecologic cancers. For cervical cancer, it refers to cancer that invades beyond the cervix, but not to the pelvic wall or lower third of the vagina and there is no parametrial invasion; for endometrial cancer, in the older FIGO classification stage scheme, it referred to endocervical glandular involvement only. Stage IIA for endometrial cancer has been eliminated in the most recent (2010) FIGO classification stage scheme. 